\title{
Review Article \\ Recent Advances in the Modeling of Transmission Lines Loaded with Split Ring Resonators
}

\author{
Jordi Naqui, Lijuan Su, Javier Mata, and Ferran Martín \\ GEMMA/CIMITEC, Departament d'Enginyeria Electrònica, Universitat Autònoma de Barcelona, 08193 Bellaterra, Spain \\ Correspondence should be addressed to Jordi Naqui; jordi.naqui@uab.cat
}

Received 19 December 2014; Accepted 23 April 2015

Academic Editor: Ivan D. Rukhlenko

Copyright (C) 2015 Jordi Naqui et al. This is an open access article distributed under the Creative Commons Attribution License, which permits unrestricted use, distribution, and reproduction in any medium, provided the original work is properly cited.

\begin{abstract}
This paper reviews the recent advances in the modeling of transmission lines loaded with split ring resonators (SRRs). It is well known that these artificial lines can exhibit a negative effective permeability in a narrow band above the SRR fundamental resonance, providing stopband functionality. By introducing shunt inductive elements to the line, the stopband can be switched to a pass band with left-handed (LH) wave propagation. For the design of microwave circuits based on these artificial lines, accurate circuit models are necessary. The former circuit model of SRR-loaded lines was presented more than one decade ago and is valid under restrictive conditions. This paper presents the progress achieved in the modeling of these artificial lines during the last years. The analysis, restricted to coplanar waveguide (CPW) transmission lines loaded only with SRRs (negative permeability transmission lines), includes the effects of SRR orientation, the coupling between adjacent resonators, and the coupling between the two SRRs constituting the unit cell. The proposed circuit models are validated through electromagnetic simulation and experimental data. It is also pointed out that the analysis can be easily extended to negative permittivity transmission lines based on complementary split ring resonators (CSRRs).
\end{abstract}

\section{Introduction}

The topic of metamaterials has attracted the interest of researchers since 2000 when the first left-handed (LH) metamaterial structure was synthesized [1]. These artificial media exhibit unusual electromagnetic properties, derived from the simultaneous negative effective permeability and permittivity, as predicted by Veselago in 1968 [2]. After the seminal work [1], various papers have experimentally confirmed the properties of LH media. Among them, the experimental verification of negative refraction [3] and the demonstration of backward leaky wave radiation in one-dimensional LH media, that is, the analogous of backward Cherenkov radiation in bulk LH media, are worth mentioning [4]. There are many books devoted to the topic of metamaterials among the available literature [5-13]. The unusual (or exotic) properties of metamaterials can be applied to the design of microwave circuits and antennas. In particular, by replacing the ordinary transmission lines in distributed circuits by artificial lines based on metamaterial concepts, it is possible to implement microwave devices with reduced size, enhanced performance, and novel functionalities on the basis of impedance and dispersion engineering $[5,6,8]$. These artificial lines based on (or inspired by) metamaterials have been designated as metamaterial transmission lines, consisting of a host line loaded with reactive elements (inductors, capacitors, and/or resonators), and can be considered to be one-dimensional metamaterials (sometimes these lines are also referred to as transmission line metamaterials [14-16]). By loading appropriately the host lines, it is possible to achieve negative effective permeability $\left(\mu_{\text {eff }}<0\right)$, permittivity $\left(\varepsilon_{\text {eff }}<0\right)$, or both negative parameters simultaneously $\left(\mu_{\mathrm{eff}}, \varepsilon_{\mathrm{eff}}<0\right)$. (Despite the fact that the permittivity and permeability are parameters of bulk media, the effective permeability and permittivity in transmission lines can be defined from the equivalence between plane wave propagation in source-free, isotropic, linear, and homogenous media and TEM wave propagation in transmission lines. The wave equations are identical if the permeability and permittivity are given by $Z_{s}^{\prime}(\omega)=j \omega \mu_{\mathrm{eff}}$ and $Y_{p}^{\prime}(\omega)=j \omega \varepsilon_{\mathrm{eff}}$, respectively, where $Z_{s}^{\prime}$ and $Y_{p}^{\prime}$ are the per-unit-length impedance (series branch) 
and admittance (shunt branch), respectively, of the equivalent T- or $\pi$-circuit model (unit cell) of the considered line.) In the last case, wave propagation is allowed, and it is backward (i.e., the phase and group velocities are antiparallel). If only one of the constitutive parameters is negative, wave propagation is not allowed. One of the most interesting properties of metamaterials and metamaterial transmission lines is the controllability (of course within certain limits) of the effective permeability and permittivity. In metamaterial transmission lines, this controllability is equivalent to the controllability of the characteristic impedance and dispersion (or phase constant), which are the parameters of interest in circuit or antenna design.

From the unit cell model of the considered metamaterial transmission line, the characteristic impedance and dispersion are given by [17]

$$
\begin{aligned}
Z_{B}^{ \pm} & =-\frac{2 B}{A-D \mp \sqrt{(A+D)^{2}-4}}, \\
\cosh (\gamma l) & =\frac{A+D}{2},
\end{aligned}
$$

where, $A, B$, and $D$ are elements of the $A B C D$ matrix of the unit cell, $l$ is the unit cell length, and $\gamma$ is the complex propagation constant; that is, $\gamma=\alpha+j \beta$, where $\alpha$ and $\beta$ are the attenuation and the phase constant, respectively. If the unit cell is symmetric with regard to the ports and it is described by a T-model, the previous expressions can be written as

$$
\begin{aligned}
Z_{B, \mathrm{~T}} & =\sqrt{Z_{s}\left(Z_{s}+2 Z_{p}\right)}, \\
\cosh (\gamma l) & =1+\frac{Z_{s}}{Z_{p}},
\end{aligned}
$$

whereas if the considered model is the $\pi$-model, the dispersion is given by the same expression, and the characteristic impedance is

$$
Z_{B, \pi}(\omega)=\sqrt{\frac{Z_{s}(\omega) Z_{p}(\omega) / 2}{1+Z_{s}(\omega) / 2 Z_{p}(\omega)}},
$$

where $Z_{s}=j \chi_{s}$ and $Z_{p}=1 / j B_{p}$ are the impedance of the series and shunt branch, respectively, of the T- or $\pi$ circuit models, with $\chi_{s}$ and $B_{p}$ being the series reactance and shunt susceptance, respectively (it is assumed that losses are negligible).

In the propagation regions, $\alpha=0$ and $\beta \neq 0$, and the dispersion relation in those regions can be expressed as

$$
\cos (\beta l)=1+\frac{Z_{s}}{Z_{p}}=1-\chi_{s} B_{p} .
$$

According to the dispersion relation, a necessary (although not sufficient) condition for wave propagation is an identical sign for the series reactance $\left(\chi_{s}\right)$ and shunt susceptance $\left(B_{p}\right)$. Moreover, if both signs are positive, wave propagation is forward, whereas it is backward (LH) if the signs of $\chi_{s}$ and $B_{p}$ are negative. To demonstrate this, we calculate the product $(\beta l) \cdot d(\beta l) / d \omega$ using expression (4). The following result is obtained:

$$
(\beta l) \cdot \frac{d(\beta l)}{d \omega}=\frac{\beta l}{\sin (\beta l)}\left(\chi_{s} \frac{d B_{p}}{d \omega}+B_{p} \frac{d \chi_{s}}{d \omega}\right) .
$$

Since, according to the Foster reactance theorem, the slope of the reactance/susceptance of a lossless network must be always positive, it follows that if $\chi_{s}, B_{p}>0$, then $\beta l$. $d(\beta l) / d \omega>0$, corresponding to forward wave propagation. Conversely, if $\chi_{s}, B_{p}<0$, then $\beta l \cdot d(\beta l) / d \omega<0$, and wave propagation is backward (note that $(\beta l) / \sin (\beta l)>0$ ).

Depending on the complexity of the loading elements of the host line, it is possible to achieve not only either forward or backward wave transmission but also a composite right/left-handed (CRLH) behavior, namely, LH wave propagation at certain frequency bands and forward (or right-handed $(\mathrm{RH})$ ) wave propagation in other frequency regions. There are two main approaches for the implementation of metamaterial transmission lines: (i) the CL-loaded approach [14-16] and (ii) the resonant-type approach $[18,19]$. In the first case, the host line is loaded with series capacitors and shunt inductors. At low frequencies, the loading elements are dominant and wave propagation is backward; at high frequencies, the host line dominates over the loading elements, and wave propagation is forward. Thus CL-loaded lines actually exhibit a CRLH behavior. In the resonant-type approach, a host line is loaded with electrically small resonators, such as split ring resonators (SRRs) [20], or complementary split ring resonators (CSRRs) [21] and other reactive elements. CPWs loaded with pairs of SRRs and shunt inductive elements exhibit a CRLH behavior [18]. Similarly, microstrip lines loaded with CSRRs and series capacitive gaps support the propagation of backward (low frequencies) and forward (high frequencies) waves [22]. Obviously, CRLH lines can also be implemented by combining the CL-loaded and the resonant-type approach (hybrid approach [23]) or by using multiple reactive elements and/or resonators in many different configurations loading the host line. By increasing the degrees of freedom, it is possible to obtain circuit functionalities not easily achievable by using ordinary lines, for example, the multiband functionality attainable by means of generalized (or extended) metamaterial transmission lines [24-27].

In this paper, the focus is on the modeling of metamaterial transmission lines based on SRRs. The aim is to analyze in detail some effects related to the resonant elements (SRRs) which are not usually taken into account but that may be important under certain conditions, namely, the coupling between SRRs and the SRR orientation with regard to the transmission line. For this reason, the analysis is restricted to SRR-loaded lines without the presence of shunt inductive elements or other elements loading the line. Thus, the considered structures exhibit stopband functionality and provided that the number of unit cells is high enough, such structures are one-dimensional negative permeability metamaterials. These structures have found applications as bandstop filters [28], multiband devices, including dualband matching networks [29] and dual-band printed dipole antennas [30] and sensors [31]. 


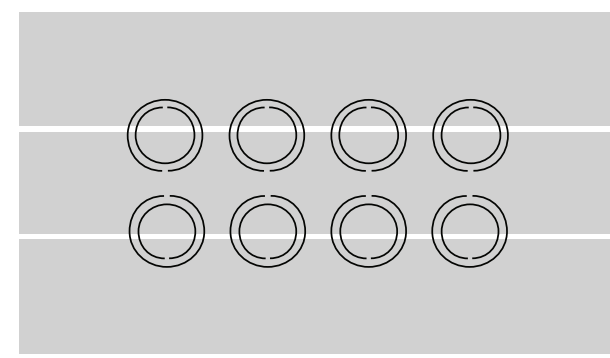

(a)

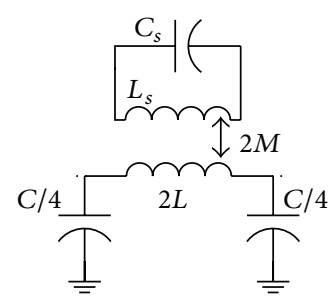

(b)

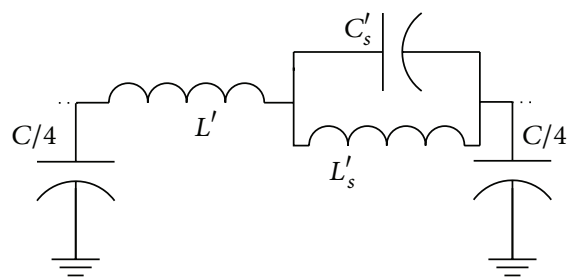

(c)

FIgURE 1: Typical topology (a) and circuit model (b) of a CPW loaded with SRRs. The model of (b) can be transformed to the one depicted in (c). The magnetic wall concept has been considered.

\section{Structure under Study and Former Circuit Model}

The typical topology of an SRR-loaded metamaterial transmission line, based on CPW technology, is depicted in Figure 1(a). The line is loaded with an array of SRR pairs etched in the back substrate side, with their centers roughly aligned with the slots of the line and their symmetry planes orthogonal to the line axis. With this configuration, the magnetic field generated by the line is able to excite the SRRs, and the structure exhibits a stopband in the vicinity of the SRR fundamental resonance frequency. This stopband has been interpreted as due to the negative effective permeability of the line just above the SRR resonance and to the high positive permeability below it (causing a strong mismatch); that is, the effective permeability is resonant and is described by the Lorentz model [1]. However, the stopband can also be explained from the lumped element equivalent circuit model of the unit cell of the structure, depicted in Figure 1(b). According to this model, firstly reported in [18], the SRRs are described by the resonant tank $L_{s}-C_{s}$; the CPW line section is described by the series inductance $L$ and the shunt capacitance $C$, and $M$ accounts for the magnetic coupling between the line and the SRRs. This model can be transformed to the model depicted in Figure 1(c), where the following transformations apply

$$
\begin{aligned}
C_{s}^{\prime} & =\frac{L_{s}}{4 M^{2} \omega_{o}^{2}}, \\
L_{s}^{\prime} & =4 M^{2} C_{s} \omega_{o}^{2}, \\
L^{\prime} & =2 L-L_{s}^{\prime}
\end{aligned}
$$

with

$$
\omega_{o}^{2}=\frac{1}{L_{s} C_{s}}=\frac{1}{L_{s}^{\prime} C_{s}^{\prime}} .
$$

It should be noted that interresonator coupling (i.e., the coupling between resonators of different cells as well as between the pair of resonators forming the unit cell) is neglected in that model.

According to the model of Figure 1, a transmission zero (notch) is expected at the resonance frequency of the SRRs.
Since the presence of many SRR pairs tends to obscure the position of the transmission zero, it is convenient to consider a single cell structure, like the one shown in Figure 2(a). The simulated response (using the Agilent Momentum commercial software) is depicted in Figure 2(b). This response can be used to extract the parameters of the circuit model of Figure 1(c), following the procedure reported in [32]. The extracted circuit elements are those indicated in the caption of Figure 2. By using these reactive parameters, the circuit response can be inferred. The results, also depicted in Figure 2(b) [33], validate the circuit model of Figure 1 (note that the agreement between the circuit and electromagnetic simulation is very good).

The model of Figure 2 provides accurate results of the electromagnetic response of SRR-loaded lines. However, this model includes neither the coupling between SRRs (potentially possible when the resonators are closely spaced) nor the effects of SRR rotation (with regard to the topology shown in Figures 1 and 2). In the next section, interresonator coupling and its effects on the response of the structures are studied in detail on the basis of more elaborated circuit models that incorporate such electromagnetic interaction. Afterwards, the analysis of SRR-loaded CPW structures with arbitrarily oriented SRRs is carried out, and it is demonstrated that mixed (electric and magnetic) coupling between the line and the resonators must be considered for an accurate description of the structures.

\section{Interresonator Coupling}

With a view to studying interresonator coupling, let us consider the SRR orientation of Figures 1 and 2, namely, with the symmetry plane of the SRR orthogonal to the line axis. As is well known, if the structure is made of multiple unit cells, coupling between resonators belonging to different cells may exist. In addition, when the SRRs of the same unit cell are close to one another, interresonator coupling within the same unit cell may also arise. Therefore, there are two possible coupling contributions between the resonant elements: (i) interunit cell coupling and (ii) intraunit cell coupling. Both couplings will be studied, assuming that the coupling type is magnetic. Note that the magnetic coupling between coplanar SRRs of adjacent cells is negative $[8,12]$, whereas that between 


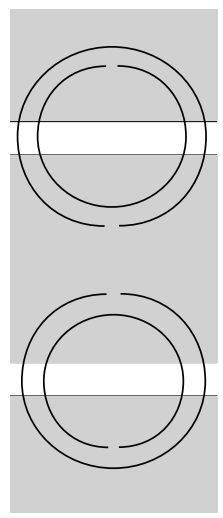

(a)

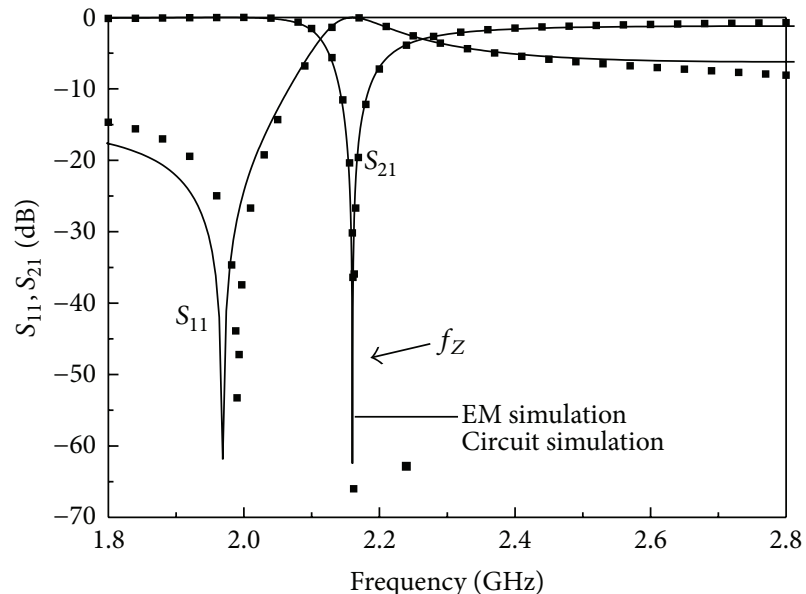

(b)

FIGURE 2: Layout (a) and electromagnetic response (b) of an SRR-loaded CPW unit cell. The considered substrate throughout this paper is Rogers $R O 3010$ with dielectric constant $\varepsilon_{r}=11.2$ and thickness $h=1.27 \mathrm{~mm}$. CPW dimensions are central strip width $W=8 \mathrm{~mm}$, width of the slots $G=1.43 \mathrm{~mm}$, and length of the line $l=8 \mathrm{~mm}$. SRR dimensions are outer ring width $c_{\text {out }}=0.36 \mathrm{~mm}$, inner ring width $c_{\text {inn }}=0.37 \mathrm{~mm}$, distance between the rings $d=0.24 \mathrm{~mm}$, and internal radius $r=2.69 \mathrm{~mm}$. The extracted circuit parameters are $L^{\prime}=1.91 \mathrm{nH}, C=2.07 \mathrm{pF}$, $L_{s}^{\prime}=0.24 \mathrm{nH}$, and $C_{s}^{\prime}=23.01 \mathrm{pF}$. From [33], reprinted with permission.

SRRs is positive [34]. The proper modeling of the magnetic coupling sign is mandatory; that is, the sign cannot be disregarded. Otherwise, the frequency response predicted by the circuit will not be able to describe correctly the behavior of the SRR-loaded lines. Let us now study these coupling mechanisms separately.

3.1. Interunit Cell Coupling. Let us consider that the magnetic coupling between adjacent resonators is accounted for by means of mutual inductances denoted as $M_{R}$. The circuit model is therefore the one depicted in Figure 3(a) [34]. In this model, losses are neglected, and the nearestneighbor approximation in the interunit cell coupling is considered, so that interresonator interaction is modeled only between adjacent resonant elements. By using the Tcircuit equivalence of magnetically coupled inductors, the circuit model of Figure 3(a) can be redrawn as depicted in Figure 3(b), from which it is possible to identify the fourport unit cell of Figure 3(c). Qualitatively, this unit cell is composed of a conventional transmission line-type circuit magnetically coupled to a secondary propagating structure. The latter structure supports a kind of backward waves that have been studied in depth in [35-38] and have been called magnetoinductive waves (MIWs). MIWs propagate within a narrow frequency band in the vicinity of SRR resonance, and the associated bandwidth is dependent on how strongly the resonators are coupled to each other. The stronger the interaction between the resonant elements, the wider the passband of the MIWs. Since multiconductor theory predicts that the resulting unit cell can propagate two modes [39], forward and backward waves are expected to coexist at some frequency band.

For the purpose of obtaining the dispersion characteristics of these SRR-loaded lines with interresonator coupling, we apply Bloch mode analysis to the four-port circuit of Figure 3(c). Let us denote $V_{\mathrm{L} i}$ and $I_{\mathrm{L} i}$ as the voltages and currents at the ports $(i=1,2)$ of the left-hand side of the unit cell and $V_{\mathrm{R} i}$ and $I_{\mathrm{R} i}$ as the variables at the right-hand side ports. The variables at both sides of the network are linked through a generalized order- 4 transfer matrix, according to

$$
\left(\begin{array}{c}
V_{L} \\
I_{L}
\end{array}\right)=\left(\begin{array}{ll}
A & B \\
C & D
\end{array}\right)\left(\begin{array}{c}
V_{R} \\
I_{R}
\end{array}\right)
$$

where $V_{L}, I_{L}, V_{R}$, and $I_{R}$ are column vectors composed of the pair of port variables, and $\mathbf{A}, \mathbf{B}, \mathbf{C}$, and $\mathbf{D}$ are order-2 matrices. The dispersion relation is obtained from the eigenmodes of the system (8); that is

$$
\operatorname{det}\left(\begin{array}{cc}
\mathbf{A}-e^{\gamma l} \cdot \mathbf{I} & \mathbf{B} \\
\mathbf{C} & \mathbf{D}-e^{\gamma l} \cdot \mathbf{I}
\end{array}\right)=0,
$$

where I is the identity matrix and the propagation factor $e^{\gamma l}$ is the eigenvalue. For reciprocal, lossless, and symmetric networks, the eigenvalues can be simplified to the solutions of $[40,41]$ :

$$
\operatorname{det}(\mathbf{A}-\cosh (\gamma l) \cdot \mathbf{I})=0,
$$

which gives

$$
\begin{aligned}
& \cosh (\gamma l) \\
& =\frac{1}{2}\left(A_{11}+A_{22} \pm \sqrt{\left(A_{11}-A_{22}\right)^{2}+4 A_{12} A_{21}}\right)
\end{aligned}
$$




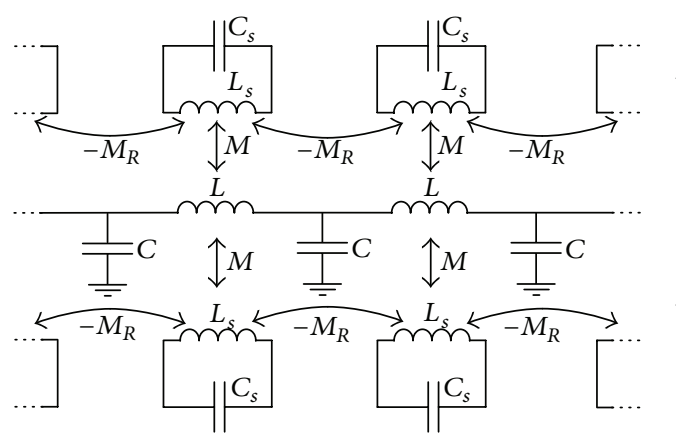

(a)

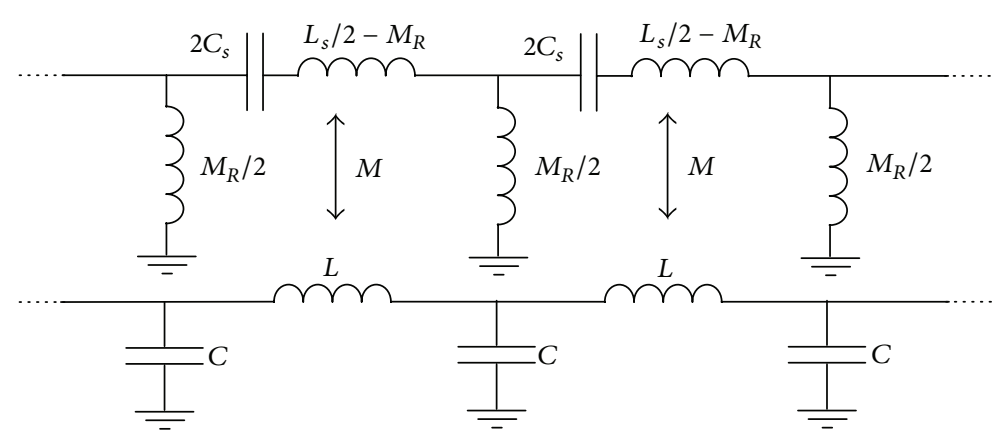

(b)

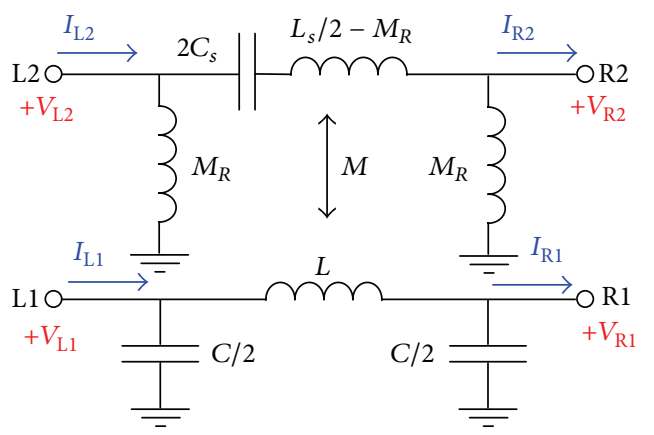

(c)

FIGURE 3: Lumped element equivalent circuit model of a periodic structure composed of a cascade of SRR-loaded CPW unit cells, including magnetic coupling between resonators of adjacent cells; model with mutual inductance between resonators (a), transformed model (b), and four-port unit cell model indicating the port voltages and currents relative to the calculation of the transfer $A B C D$ matrix. From [34], reprinted with permission.

where the elements of $\mathbf{A}$ matrix (inferred from the network of Figure 3(c)) are [34]

$$
\mathbf{A}=\mathbf{D}^{t}=\left(\begin{array}{cc}
1-\frac{L C}{2} \omega^{2} & \frac{M}{M_{R}} \\
-\frac{M C}{2} \omega^{2} & \frac{1}{2 M_{R}}\left(L_{s}-\frac{1}{C_{s} \omega^{2}}\right)
\end{array}\right) .
$$

Since the network of Figure 3(c) is lossless, the elements of $\mathbf{A}\left(A_{i j}\right)$ are real numbers. Hence, if the radicand of the square root in (11) is positive, the propagation constant is either purely real $(\alpha \neq 0, \beta=0)$ or purely imaginary $(\alpha=0$, $\beta \neq 0$ ), corresponding to evanescent or propagating modes, respectively. However, if the radicand in (11) is negative, the two solutions are of the form $\gamma=\alpha \pm j \beta$, corresponding to complex modes [42]. The frequency band that supports complex modes is thus obtained by forcing the radicand in (11) to be negative. Since complex modes do not carry net power, the frequency band supporting such modes is a rejection band, despite being of different nature from that associated to evanescent modes (where $\alpha \neq 0, \beta=0$ ).

Inspection of (11) and (12) reveals that a necessary condition for the presence of complex modes is the fact that $M$ is different from zero (this is always the case, unless the substrate of the considered CPW is extremely thick). The two modal solutions for $M=0$ (meaning that the host line and the SRR array are decoupled) are simply the dispersion relation of a lossless transmission line described by the wellknown LC ladder network and the dispersion of an array of

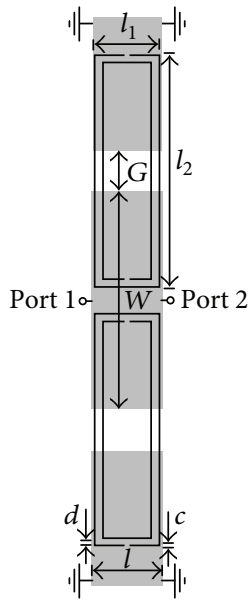

FIGURE 4: Unit cell of a CPW transmission line loaded with a pair of SRRs designed to enhance coupling between resonators of neighboring cells. Dimensions are $W=9.1 \mathrm{~mm}, G=1.7 \mathrm{~mm}$, $l=3 \mathrm{~mm}, c=d=0.15 \mathrm{~mm}, l_{1}=2.8 \mathrm{~mm}$, and $l_{2}=9.8 \mathrm{~mm}$. The Bloch waves propagate from port 1 to port 2. From [34], reprinted with permission.

inductively (edge) coupled SRRs, where backward MIWs in a narrow frequency band in the vicinity of SRR resonance are supported [34].

To validate the model of Figure 3, the unit cell of Figure 4 has been considered [34]. In this unit cell, the resonators 

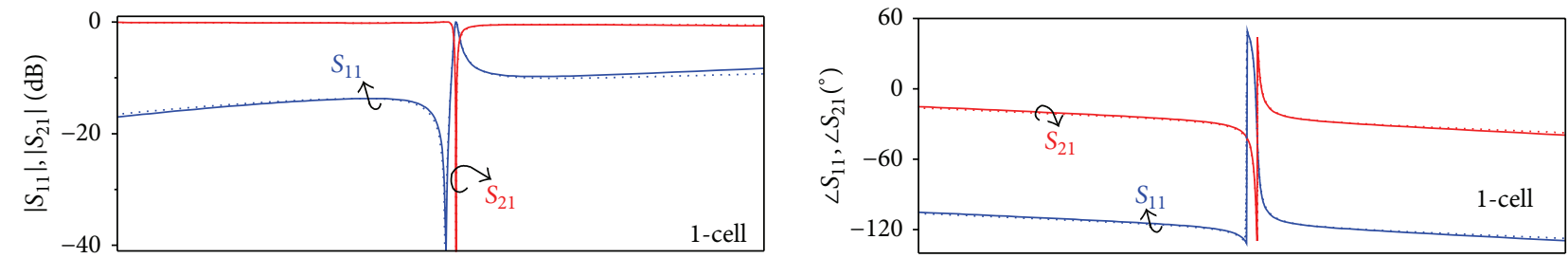

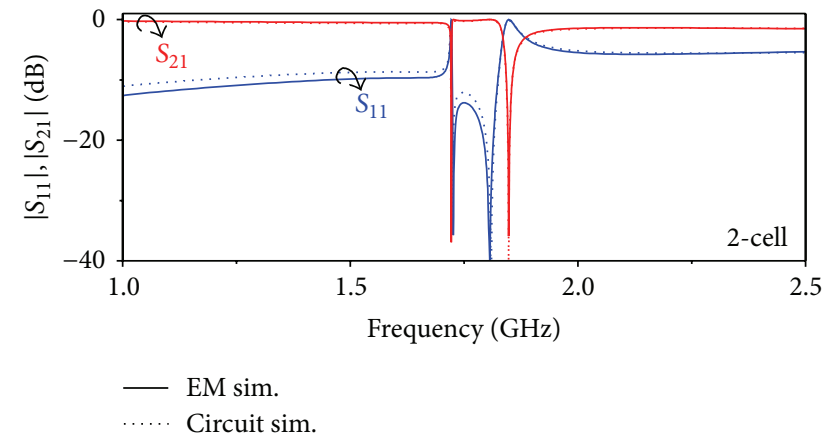

(a)

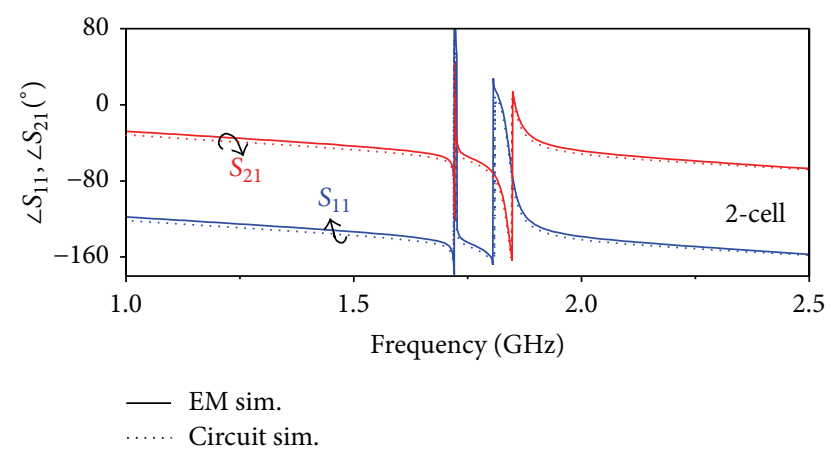

(b)

Figure 5: Magnitude (a) and phase (b) of the lossless transmission $\left(S_{21}\right)$ and reflection $\left(S_{11}\right)$ coefficients for a unit cell and for two cascaded unit cells of the structure in Figure 4. The extracted circuit parameters are $L=1.01 \mathrm{nH}, C=1.40 \mathrm{pF}, L_{s}=17.66 \mathrm{nH}, C_{s}=0.45 \mathrm{pF}, M=0.72 \mathrm{nH}$, and $M_{R}=1.17 \mathrm{nH}$. From [34], reprinted with permission.

are rectangular in order to enhance interresonator coupling. The circuit parameters have been extracted according to the general method reported in [32] (which ignores interresonator coupling) and the specific procedure (to determine $M_{R}$ ) thoroughly described in [34]. Figure 5 depicts the circuit and electromagnetic simulations of the frequency response of a single unit cell and two unit cells, where the good agreement between circuit and electromagnetic simulations can be appreciated. The well-known frequency-splitting phenomenon as a consequence of the interresonator coupling should be noticed.

Once the circuit parameters have been extracted, the pair of modal propagation constants given by (11) can be inferred. The results are depicted in Figure 6. In the first allowed band there is a region with bi-valued propagation constant: one (forward) corresponding to transmission-line type propagation and the other (backward) related to magnetoinductive waves. Then, a region with a pair of conjugate complex propagation constants (complex modes) appears where forward and backward waves interfere with each other, followed by a region of evanescent waves. Finally, a forward wave transmission band emerges again. Hence, the enhancement of the stopband due to interresonator coupling is explained by the appearance of complex waves in the lower frequency region of that stopband (the complex modes exist from $1.843 \mathrm{GHz}$ to $1.961 \mathrm{GHz}$, and the evanescent modes extend up to $1.977 \mathrm{GHz}$ ). However, the magnetic coupling between SRRs of adjacent cells is limited in practice and, hence, bandwidth broadening is also limited. The dispersion relation of a periodic structure composed of a cascade of the unit cells of Figure 4, obtained by means of the full-wave eigenmode solver of CST Microwave Studio, is also depicted in Figure 6. There is good agreement with the analytical
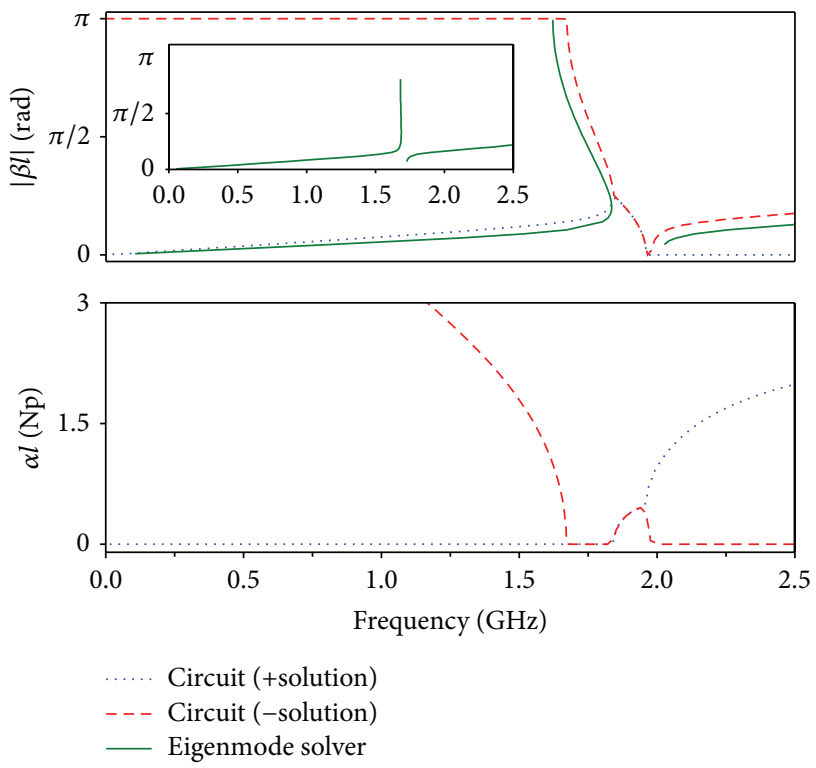

FIGURE 6: Dispersion relation for the structure of Figure 4 inferred from an eigenmode solver and from its equivalent circuit model of Figure 3(c). The circuit parameters are those indicated in the caption of Figure 5. The dispersion relation for the structure of Figure 4 with $l=4.8 \mathrm{~mm}$ is depicted in the inset. The attenuation constant $\alpha$ is not provided by the eigenmode solver. From [34], reprinted with permission.

dispersion curve predicted by the circuit model. That is, the bi-valued region is perfectly predicted by the eigenmode solver. Nevertheless, since there is no electromagnetic field pattern with net current transfer in the stopband, the tool is 


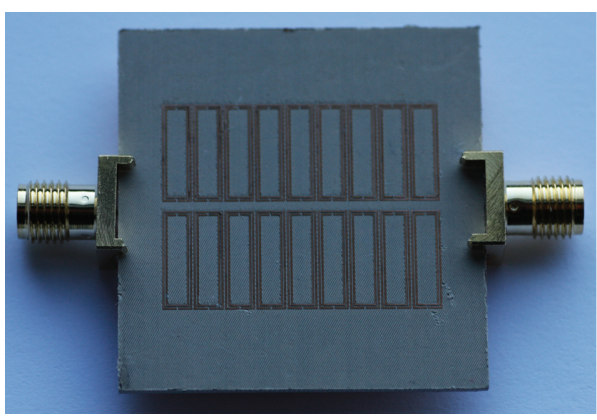

(a)

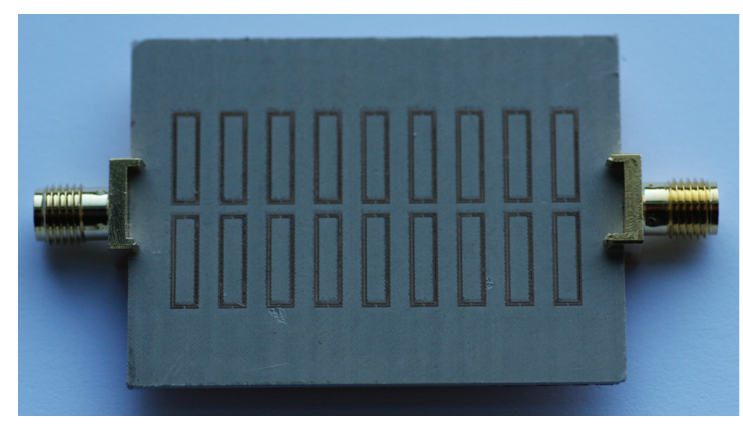

(b)

FIGURE 7: Photograph of the bottom face of the fabricated order-9 structures composed of the unit cell in Figure 4 with (a) $l=3 \mathrm{~mm}$ and (b) $l=4.8 \mathrm{~mm}$. From [34], reprinted with permission.
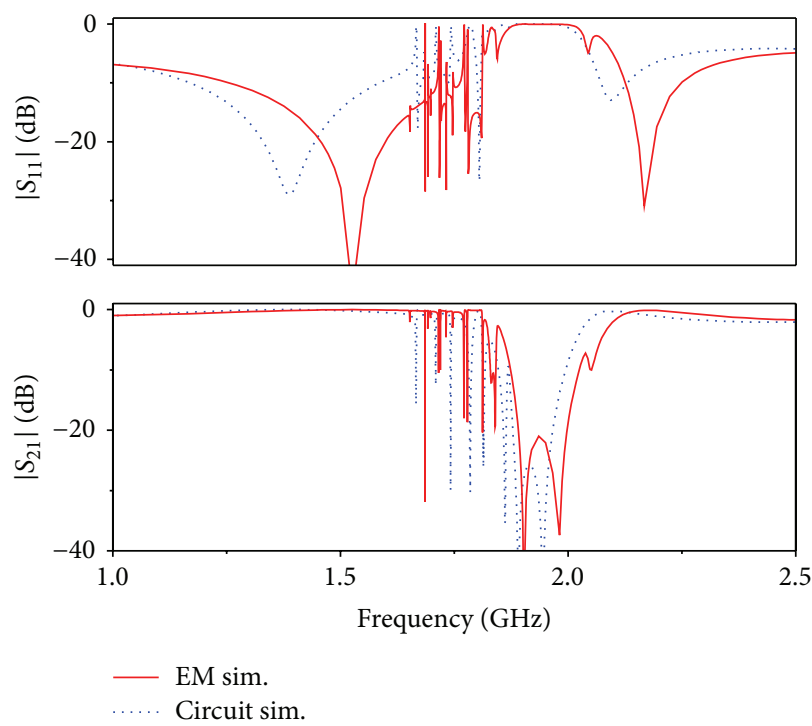

(a)
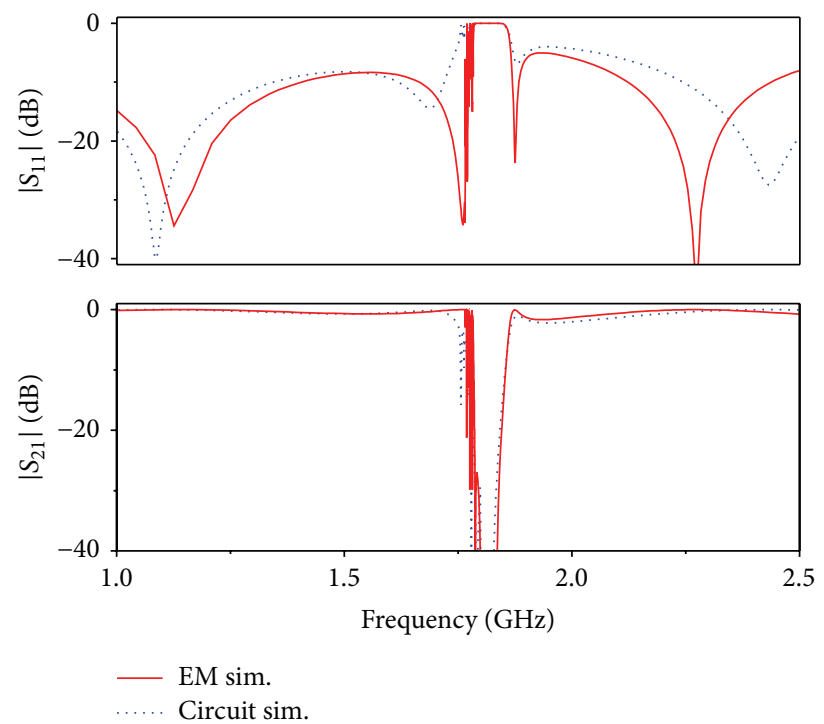

(b)

FIGURE 8: Lossless electromagnetic and circuit simulations of the transmission and reflection coefficients (magnitude) for nine cascaded cells of the structure in Figure 4 for (a) $l=3 \mathrm{~mm}$ and (b) $l=4.8 \mathrm{~mm}$ (the interresonator separation has been increased from $0.2 \mathrm{~mm}$ to $2 \mathrm{~mm}$ ). The circuit parameters are those indicated in the caption of Figure 5, with the exception of $M_{R}=0.22 \mathrm{nH}$ in (b). Furthermore, in (b), a $\mathrm{CPW}$ section of $1.8 \mathrm{~mm}$ length has been cascaded between the port R1 and the port L1 of the contiguous cell in the circuit model. From [34], reprinted with permission.

not able to provide the dispersion curves in that region. For comparison purposes, we have also considered a structure whose resonators are spaced further apart providing much weaker coupling. The dispersion diagram, depicted in the inset of Figure 6, reveals that the stopband bandwidth is significantly narrower. Therefore, the dispersion diagrams indicate that most of the stopband in the structure of Figure 4 is related to the presence of complex modes rather than evanescent modes. In other words, as long as interresonator coupling is significant, complex modes may be the dominant mechanism of signal rejection (in the vicinity of SRR fundamental resonance) of these SRR-loaded structures.

The experimental validation has been carried out by considering two 9-cell structures, one with $l=3$ and the other one with $l=4.8$ (corresponding to tightly and weakly coupled resonators, resp.), in coherence with the two considered structures of which the dispersion relation was determined. The photographs of these SRR-loaded lines are shown in Figure 7. Figure 8 shows the comparison between the lossless electromagnetic simulation and circuit simulation for both structures, whereas Figure 9 compares the lossy electromagnetic simulation and measurement. As expected, interresonator coupling enhances the stopband bandwidth. The measured stopbands are also in accordance with those obtained from the dispersion relation. Hence, the dispersion relation inferred from the multiterminal circuit model is a powerful tool to gain insight into the stopband and the effects of interresonator coupling in SRR-loaded lines.

3.2. Intraunit Cell Coupling. In the previous subsection, the coupling between the two resonators of the unit cell was neglected, and this is reasonable if the resonators are not 

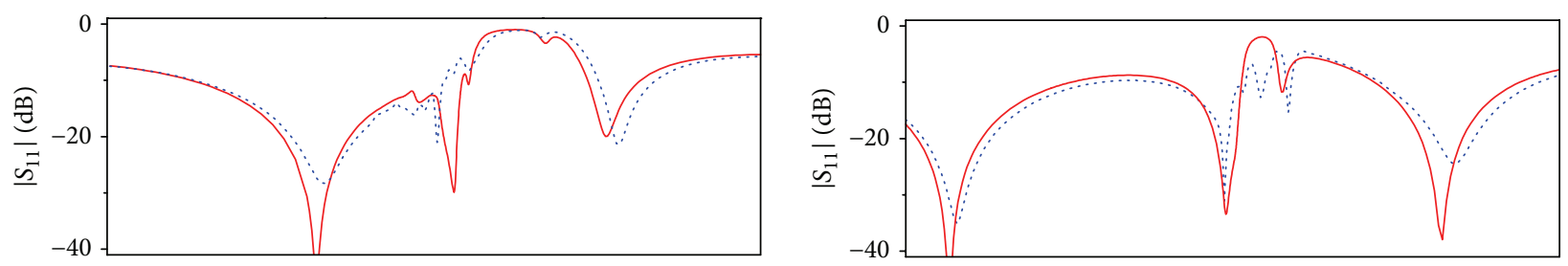

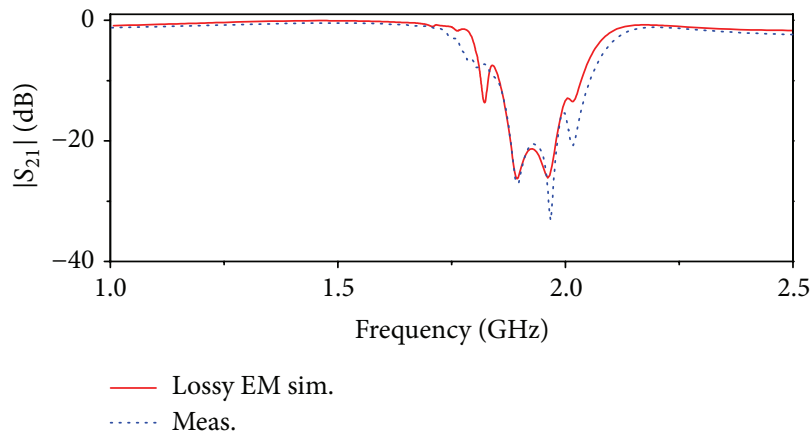

(a)

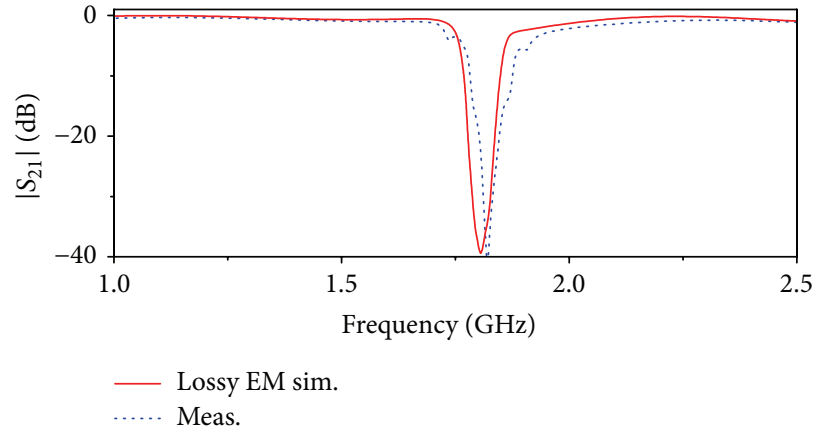

(b)

FIGURE 9: Measurement and lossy electromagnetic simulation of the transmission and reflection coefficients of the structures in Figure 7; (a) $l=3 \mathrm{~mm}$ and (b) $l=4.8 \mathrm{~mm}$. The loss tangent in Rogers $R O 3010$ is $\tan \delta=0.0023$. From [34], reprinted with permission.

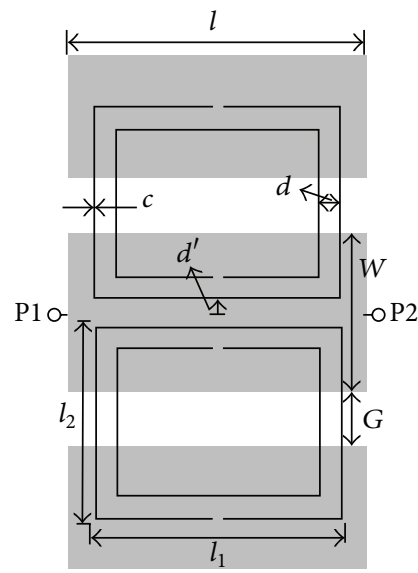

(a)

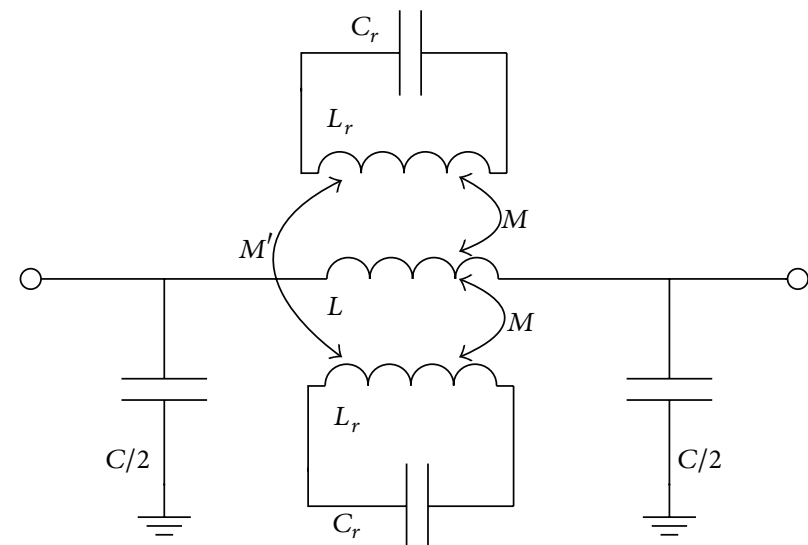

(b)

FIGURE 10: Typical topology of a CPW loaded with a pair of square-shaped SRRs (a) and lumped element equivalent circuit model considering magnetic coupling between SRRs (b).

closely spaced. If this is not the case, as is in general, interresonator coupling emerges. To gain insight into the effects of such a coupling, we consider the structure in Figure 10(a) where the interresonator magnetic coupling is included in the circuit model of the unit cell (through $M^{\prime}$ ), whilst the coupling between adjacent resonators is neglected (i.e., $M_{R}=0$ ). The resulting model (unit cell) is depicted in Figure 10(b) [43] (for coherence with [43], $L_{r}$ and $C_{r}$ stand for the SRR inductance and capacitance, resp.). The magnetic coupling between the resonators of the unit cell shifts the transmission zero frequency as compared to the structure with negligible coupling. To demonstrate this, it suffices to apply the T-circuit equivalence of a pair of magnetically coupled inductors (Figure 11(a)) [44]. Since there is a magnetic wall across the symmetry plane of the structure, it follows that the circuit model of Figure 10(b) can be transformed as shown in Figure 11(b), and by applying the magnetic wall concept, the circuit of Figure 11(c) results. By comparing this latter circuit model to the one depicted in Figure 1(b), it is clear that $M^{\prime}$ modifies the transmission zero frequency, which is now given by

$$
\omega_{o}^{\prime}=\frac{\omega_{o}}{\sqrt{1+M^{\prime} / L_{r}}}=\frac{\omega_{o}}{\sqrt{1+k_{m}}},
$$

where $k_{m}$ is the magnetic coupling coefficient between the resonators [44] and $\omega_{o}$ is the intrinsic resonance frequency of the SRRs, defined in (7). According to the model of 


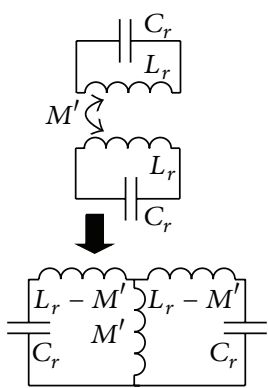

(a)

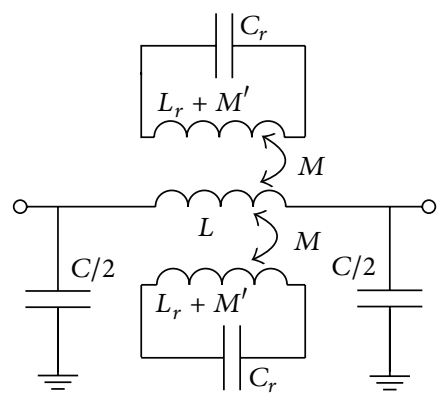

(b)

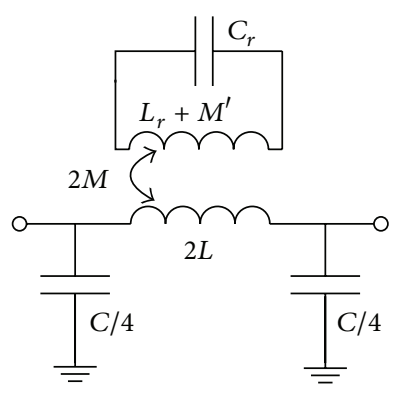

(c)

FIGURE 11: Equivalent T-circuit model of a pair of magnetically coupled inductors (a), circuit model of the structure of Figure 10(a) with the inductive coupling between resonators transformed to the equivalent T-model (b), and circuit that results by applying the magnetic wall concept (c). From [43], reprinted with permission.

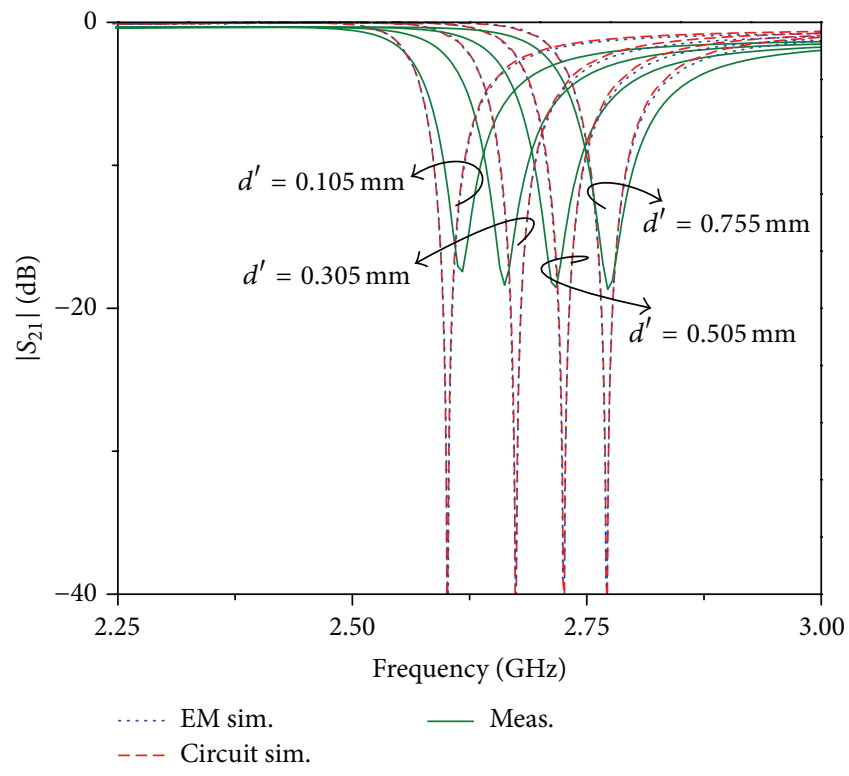

(a)

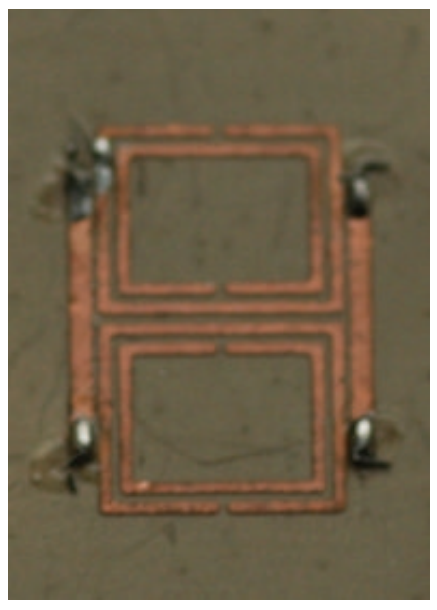

(b)

FIGURE 12: Frequency response for different values of $d^{\prime}$ (a) and detail of the bottom side of one of the fabricated samples (b). In reference to Figure $10(\mathrm{a})$, dimensions are $l_{1}=4.8 \mathrm{~mm}, l_{2}=3.8 \mathrm{~mm}, c=d=0.2 \mathrm{~mm}, l=5.6 \mathrm{~mm}, W=3 \mathrm{~mm}$, and $G=1.01 \mathrm{~mm}$. The circuit parameters are listed in Table 1. From [43], reprinted with permission.

Figure 11(c), the effects of magnetic coupling between the resonators of the unit cell can be accounted for by simply modifying the SRR inductance and considering the former circuit model of Figure 1(b). As a result of the positive sign in $M^{\prime}$, this mutual inductance shifts the resonance frequency downwards. However, this is usually ignored regardless of the distance (and hence coupling) between the SRRs. It is worth mentioning that if SRRs with different dimensions (and hence different coupling to the line) are considered, interresonator coupling also modifies the position of the transmission zero frequencies (with regard to the intrinsic resonance frequency of the individual resonators). Analytical expressions are given in [43]. Nevertheless, in contrast to the symmetric case, mutual coupling cannot be neglected in CPWs asymmetrically loaded with pairs of SRRs. Furthermore, in the most general asymmetric case, it is not easy to identify a simplified equivalent circuit similar to the one of Figure 1(c), that is, without mutual inductances.

To validate the models in Figures 10 and 11 of a CPW symmetrically loaded with a pair of magnetically coupled SRRs and the effects of coupling, four different structures with identical SRRs but with different interresonator distance have been considered. The lossless electromagnetic simulations of the structure $\left(S_{21}\right)$, assuming different values of interresonator distance, $d^{\prime}$, are depicted in Figure 12(a). The circuit parameters for the four considered cases are indicated in Table 1 . As $d^{\prime}$ increases, the mutual coupling $M^{\prime}$ decreases, and the resonance frequency increases. Note that the other circuit parameters do not significantly vary, and the agreement between circuit and electromagnetic simulations 


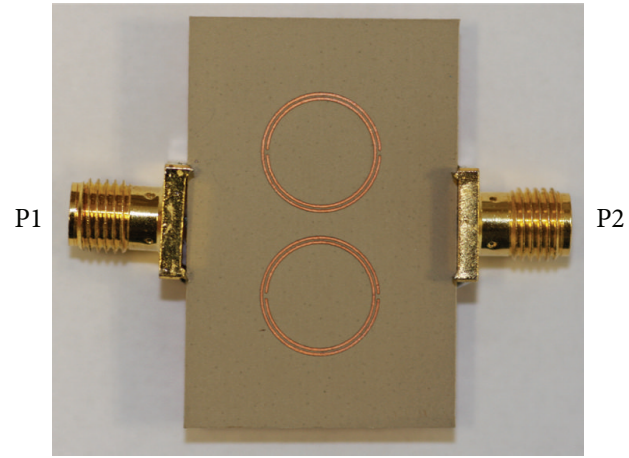

(a)

2
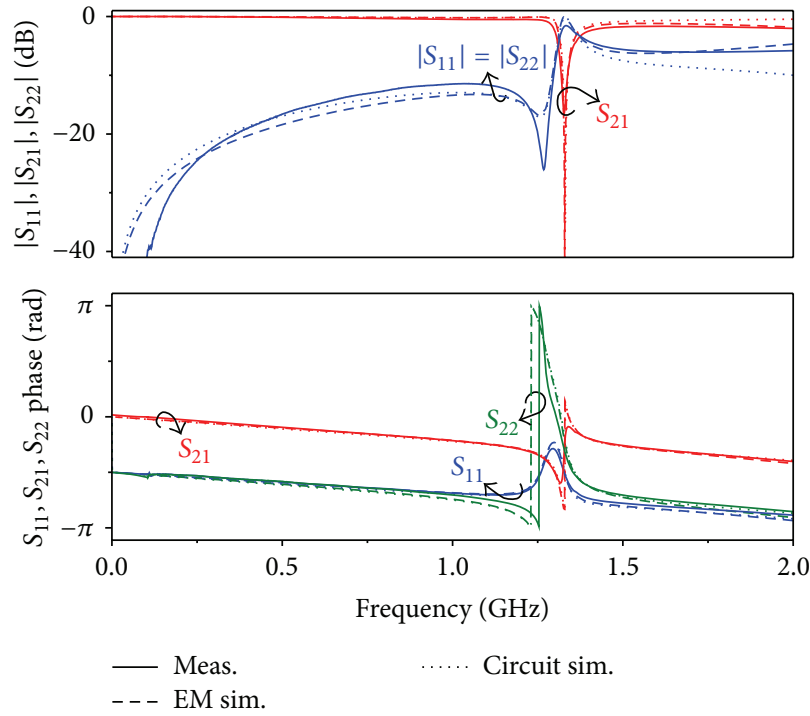

(b)

FIGURE 13: Bottom side photograph of the fabricated SRR-loaded CPW with $\phi=90^{\circ}$ (a) and frequency response (b). Dimensions are $W=$ $10.4 \mathrm{~mm}, G=1.6 \mathrm{~mm}$, external radius $r_{\text {ext }}=5 \mathrm{~mm}$, and $c=d=0.2 \mathrm{~mm}$. The extracted circuit parameters are $L=1.5 \mathrm{nH}, C=6.1 \mathrm{pF}$, $L_{s}=28.2 \mathrm{nH}, C_{s}=0.43 \mathrm{pF}, M=1.35 \mathrm{nH}$, and $C_{a}=0.08 \mathrm{pF}$. From [46], reprinted with permission.

TABLE 1: Extracted circuit parameters for different values of $d^{\prime}$.

\begin{tabular}{lcccccc}
\hline$d^{\prime}(\mathrm{mm})$ & $L(\mathrm{nH})$ & $C(\mathrm{pF})$ & $C_{r}(\mathrm{pF})$ & $L_{r}(\mathrm{nH})$ & $M(\mathrm{nH})$ & $M^{\prime}(\mathrm{nH})$ \\
\hline 0.105 & 1.82 & 1.58 & 0.44 & 6.85 & 0.82 & 1.74 \\
0.305 & 1.86 & 1.58 & 0.44 & 6.85 & 0.82 & 1.29 \\
0.505 & 1.84 & 1.57 & 0.43 & 6.85 & 0.81 & 1.02 \\
0.755 & 1.85 & 1.55 & 0.43 & 6.85 & 0.80 & 0.80 \\
\hline
\end{tabular}

in the region of interest is very good, pointing out the validity of the model. The measured responses (plotted in Figure 12(a)) are also in good agreement.

\section{SRR Rotation: Mixed Coupling}

In the SRR-loaded CPW structures considered thus far, the slits of the SRR (aligned with the symmetry plane of the particle) are orthogonally oriented to the line axis. With this configuration, the coupling mechanism between the line and the resonators is magnetic, and the circuit models are those presented in the previous sections. By contrast, let us now consider that the SRRs are oriented with an arbitrary angle $\phi$ (where $\phi=0^{\circ}$ corresponds to the orthogonal orientation). If $\phi \neq 0^{\circ}$, the SRR-loaded line is no longer symmetric with regard to the ports, and the circuit model must account for that asymmetry (note that any of the structures and circuit models previously presented are symmetric with regard to the ports). Moreover, since the SRR exhibits an electric dipole moment (at the fundamental resonance) in the plane of the particle and orthogonal to its symmetry plane $[8,45]$, it follows that, by rotating the SRR $\left(\phi \neq 0^{\circ}\right)$, electric coupling between the line and the SRR does also arise.
As a case study, consider the structure shown in Figure 13(a), where $\phi=90^{\circ}$. The response of this structure (unit cell), shown in Figure 13(b), indicates that the phases of $S_{11}$ and $S_{22}$ are different, as expected on account of the asymmetry with regard to the ports. Obviously, these different phases cannot be explained by the previous circuit models. Necessarily, the circuit model describing the SRR-loaded $\mathrm{CPW}$ with $\phi \neq 0^{\circ}$ must be asymmetric. By including electric coupling between the line and the SRRs in the model of Figure 1(b), that is, by modeling the structure through mixed magnetoelectric coupling, the resulting circuit (depicted in Figure 14) is asymmetric, and it describes very accurately the frequency response of the structure [46]. Note that the asymmetry in the circuit model (necessary to explain that $S_{11} \neq S_{22}$ ) comes from the magnetic coupling (the dot convention for the mutual inductance is used, where the currents entering dot-marked terminals produce additive magnetic fluxes), although this is not manifested if electric coupling is not present.

From the electromagnetic response depicted in Figure 13(b), the circuit parameters of the model of Figure 14 were extracted [46]. The resulting circuit parameters are indicated in the caption of Figure 13. The agreement between the circuit simulation (also depicted in Figure 13(b)) and the electromagnetic simulation is good. The device was fabricated, and the measured response is also in good accordance with the circuit and electromagnetic simulations. These results clearly point out that, to avoid electric coupling between the line and the SRRs, it is mandatory to etch the SRRs with their symmetry plane orthogonally oriented to the line axis. Otherwise, mixed coupling appears and must be taken into account for an accurate description of 


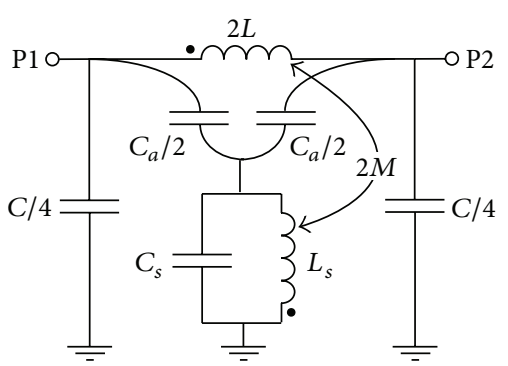

FIGURE 14: Proposed equivalent circuit model (unit cell) of the CPW loaded with arbitrarily oriented SRRs $\left(C_{a}=0\right.$ for $\left.\phi=0^{\circ}\right)$. The magnetic wall concept has been applied. From [46], reprinted with permission.

the SRR-loaded line through its lumped element equivalent circuit model.

\section{Discussion}

The description of the previous SRR-loaded lines through lumped element equivalent circuits assumes that the electrical size of the unit cell is small. Under these conditions, the proposed circuits provide an accurate description of the structures up to frequencies slightly above the SRR resonance frequency. At higher frequencies, the circuit response and the electromagnetic (or measured) response progressively diverge since the SRRs dimensions are comparable or larger than the wavelength. This effect is more pronounced if the electrical size of the SRRs is not very small, as occurs in SRRs with weakly coupled loops or in SRRs consisting of a single loop. In a very recent paper [47], the model of Figure 1(b) has been improved by representing the transmission line section by two $\pi$-cells, keeping the same number of independent parameters. This model allows for better matching with fullwave electromagnetic simulation and measurement and is especially convenient when single-loop SRRs are considered (as is the case of the structures in [47]). By describing the line section with two cascaded $\pi$-cells, more accurate results can be obtained if the SRR and hence the transmission line section of the unit cell are not electrically very small. However, the penalty, from the point of view of circuit design, is a higher complexity. In other words, the circuits proposed in [47] are justified if the considered resonators have a moderate electrical size. If the electric size of the particles is small, then the circuits proposed in the previous sections suffice.

Concerning the bandwidth enhancement caused by interunit cell coupling, this effect also manifests in SRRs operating inside waveguides or free space [48].

Lastly, it is worth mentioning that similar improvements to the original model of the SRR-loaded line of Figure 1(b) can also be applied to the original model of a microstrip line loaded with complementary split ring resonators (CSRRs), formerly reported in [19]. Thus, the effects of CSRR rotation (leading to mixed coupling) have been reported in [46], and the effects of interresonator coupling between CSRRs of adjacent cells (enhancing the stopband bandwidth) were studied in [49]. In the case of microstrip lines loaded with (electrically) coupled CSRRs, the presence of complex modes has been explained as due to the coexistence of electromagnetic and electroinductive waves [50]. Typically, microstrip lines are loaded with CSRRs including a single resonant element in the unit cell. It is for this reason that an analogous study to the one reported in Section 3.2 for CSRRloaded lines has not been considered so far.

\section{Conclusions}

In conclusion, the recent progress in the circuit modeling of SRR-loaded CPW transmission lines has been reviewed. We have modeled three main effects not considered in the former model of the unit cell presented more than one decade ago. Specifically, we have analyzed the effects of interresonator coupling between resonators of adjacent cells and between the pair of resonators of the unit cell, and we have also studied the SRR angular orientation. All these effects have been taken into account in order to accurately describe the structure through novel lumped element equivalent circuits. In brief, coupling between adjacent resonators enhances the stopband bandwidth, and this has been found to be caused by the appearance of complex modes; coupling between the two resonators of the unit cell modifies the notch frequency and can be simply modeled by modifying the inductance of the SRR. Finally, it has been found that if the SRR symmetry plane is not orthogonal to the line axis, mixed (i.e., electric and magnetic) coupling between the line and the SRRs emerges, and both coupling mechanisms must be included in the circuit model for an accurate description of the structures.

\section{Conflict of Interests}

The authors declare that there is no conflict of interests regarding the publication of this paper.

\section{Acknowledgments}

This work has been supported by MINECO-Spain (Projects TEC2010-17512 METATRANSFER, CONSOLIDER EMET CSD2008-00066, EXPLORA TEC2011-13615-E, and TEC2013-40600-R COM-SEN-RFID) and by Generalitat de Catalunya (Project 2014SGR-157). Jordi Naqui and Ferran Martín have been awarded with an FPU grant (AP2010-0431) and with an ICREA Academia Award, respectively.

\section{References}

[1] D. R. Smith, W. J. Padilla, D. C. Vier, S. C. Nemat-Nasser, and S. Schultz, "Composite medium with simultaneously negative permeability and permittivity," Physical Review Letters, vol. 84, no. 18, pp. 4184-4187, 2000.

[2] V. G. Veselago, "The electrodynamics of substances with simultaneously negative values of $\varepsilon$ and $\mu$," Soviet Physics Uspekhi, vol. 10, no. 4, pp. 509-514, 1968.

[3] R. A. Shelby, D. R. Smith, and S. Schultz, "Experimental verification of a negative index of refraction," Science, vol. 292, no. 5514, pp. 77-79, 2001. 
[4] A. Grbic and G. V. Eleftheriades, "Experimental verification of backward-wave radiation from a negative refractive index metamaterial," Journal of Applied Physics, vol. 92, no. 10, pp. 5930-5935, 2002.

[5] G. V. Eleftheriades and K. G. Balmain, Eds., Negative-Refraction Metamaterials: Fundamental Principles and Applications, John Wiley \& Sons, New York, NY, USA, 2005.

[6] C. Caloz and T. Itoh, Electromagnetic Metamaterials: Transmission Line Theory and Microwave Applications, John Wiley \& Sons, New York, NY, USA, 2006.

[7] N. Engheta and R. W. Ziolkowski, Metamaterials: Physics and Engineering Explorations, John Wiley \& Sons, New York, NY, USA, 2006.

[8] R. Marqués, F. Martín, and M. Sorolla, Metamaterials with Negative Parameters: Theory, Design and Microwave Applications, John Wiley \& Sons, New York, NY, USA, 2007.

[9] A. K. Sarychev and V. M. Shalaev, Electrodynamics of Metamaterials, World Scientific Publishing, Singapore, 2007.

[10] P. Markos and C. M. Sokoulis, Wave Propagation: From Electrons to Photonic Crystals and Left-Handed Materials, Princeton University Press, 2008.

[11] F. Capolino, Ed., Metamaterials Handbook, CRC Press, New York, NY, USA, 2009.

[12] L. Solymar and E. Shamonina, Waves in Metamaterials, Oxford University Press, Oxford, UK, 2009.

[13] T. J. Cui, D. R. Smith, and R. Liu, Eds., Metamaterials: Theory, Design and Applications, Springer, New York, NY, USA, 2010.

[14] A. K. Iyer and G. V. Eleftheriades, "Negative refractive index metamaterials supporting 2-D waves," in Proceedings of the IEEE MSS-S International Microwave Symposium Digest, pp. 412-415, Seattle, Wash, USA, June 2002.

[15] A. A. Oliner, "A periodic-structure negative-refractive-index medium without resonant elements," in Proceedings of the URSI Digest, IEEE-AP-S USNC/URSI National Radio Science Meeting, p. 41, San Antonio, Tex, USA, June 2002.

[16] C. Caloz and T. Itoh, "Application of the transmission line theory of left-handed ( $\mathrm{LH})$ materials to the realization of a microstrip LH transmission line," in Proceedings of the IEEE AP-S USNC/URSI National Radio Science Meeting, vol. 2, pp. 412-415, IEEE, San Antonio, Tex, USA, June 2002.

[17] D. M. Pozar, Microwave Engineering, Addison Wesley, New York, NY, USA, 1990.

[18] F. Martín, J. Bonache, F. Falcone, M. Sorolla, and R. Marqués, "Split ring resonator-based left-handed coplanar waveguide," Applied Physics Letters, vol. 83, no. 22, pp. 4652-4654, 2003.

[19] J. D. Baena, J. Bonache, F. Martín et al., "Equivalent-circuit models for split-ring resonators and complementary splitring resonators coupled to planar transmission lines," IEEE Transactions on Microwave Theory and Techniques, vol. 53, no. 4, pp. 1451-1460, 2005.

[20] J. B. Pendry, A. J. Holden, D. J. Robbins, and W. J. Stewart, "Magnetism from conductors and enhanced nonlinear phenomena," IEEE Transactions on Microwave Theory and Techniques, vol. 47, no. 11, pp. 2075-2084, 1999.

[21] F. Falcone, T. Lopetegi, J. D. Baena, R. Marqués, F. Martín, and M. Sorolla, "Effective negative- $\varepsilon$ stopband microstrip lines based on complementary split ring resonators," IEEE Microwave and Wireless Components Letters, vol. 14, no. 6, pp. 280-282, 2004.

[22] M. Gil, J. Bonache, J. Selga, J. García-García, and F. Martín, "Broadband resonant-type metamaterial transmission lines,"
IEEE Microwave and Wireless Components Letters, vol. 17, no. 2, pp. 97-99, 2007.

[23] J. Bonache, M. Gil, I. Gil, J. García-García, and F. Martín, "Limitations and solutions of resonant-type metamaterial transmission lines for filter applications: the hybrid approach," in Proceedings of the IEEE MTT-S International Microwave Symposium Digest, pp. 939-942, San Francisco, Calif, USA, June 2006.

[24] A. Rennings, S. Otto, J. Mosig, C. Caloz, and I. Wolff, "Extended composite right/left-handed metamaterial and its application as quadband quarter-wavelength transmission line," in Proceedings of the Asia-Pacific Microwave Conference (APMC '06), pp. 1405-1408, Yokohama, Japan, December 2006.

[25] G. V. Eleftheriades, "A generalized Negative-Refractive-Index Transmission-Line (NRI-TL) metamaterial for dual-band and quad-band applications," IEEE Microwave and Wireless Components Letters, vol. 17, no. 6, pp. 415-417, 2007.

[26] G. Sisó, M. Gil, J. Bonache, and F. Martin, "Generalized model for multiband metamaterial transmission lines," IEEE Microwave and Wireless Components Letters, vol. 18, no. 11, pp. 728-730, 2008.

[27] C. Camacho-Peñalosa, T. M. Martín-Guerrero, J. Esteban, and J. E. Page, "Derivation and general properties of artificial lossless balanced composite right/left-handed transmission lines of arbitrary order," Progress In Electromagnetics Research B, no. 13, pp. 151-169, 2009.

[28] F. Martín, F. Falcone, J. Bonache, R. Marqués, and M. Sorolla, "Miniaturized CPW stop band filters based on multiple tuned split ring resonators," IEEE Microwave and Wireless Components Letters, vol. 13, no. 12, pp. 511-513, 2003.

[29] F. Paredes, G. Zamora, J. Bonache, and F. Martín, "Dual-band impedance matching networks based on split ring resonators for applications in radiofrequency identification (RFID)," IEEE Transactions on Microwave Theory and Techniques, vol. 58, no. 5, pp. 1159-1166, 2010.

[30] F. J. Herraiz-Martínez, L. E. García-Muñoz, D. GonzálezOvejero, V. González-Posadas, and D. Segovia-Vargas, "Dualfrequency printed dipole loaded with split ring resonators," IEEE Antennas and Wireless Propagation Letters, vol. 8, pp. 137140, 2009.

[31] J. Naqui, M. Durán-Sindreu, and F. Martín, "Novel sensors based on the symmetry properties of split ring resonators (SRRs)," Sensors, vol. 11, no. 8, pp. 7545-7553, 2011.

[32] F. Aznar, M. Gil, J. Bonache et al., "Characterization of miniaturized metamaterial resonators coupled to planar transmission lines through parameter extraction," Journal of Applied Physics, vol. 104, no. 11, Article ID 114501, 8 pages, 2008.

[33] F. Aznar, M. Gil, J. Bonache, and F. Martín, "Modelling metamaterial transmission lines: a review and recent developments," Opto-Electronics Review, vol. 16, no. 3, pp. 226-236, 2008.

[34] J. Naqui, A. Fernández-Prieto, F. Mesa, F. Medina, and F. Martín, "Effects of inter-resonator coupling in split ring resonator loaded metamaterial transmission lines," Journal of Applied Physics, vol. 115, no. 19, Article ID 194903, 2014.

[35] E. Shamonina, V. A. Kalinin, K. H. Ringhofer, and L. Solymar, "Magneto-inductive waveguide," Electronics Letters, vol. 38, no. 8, pp. 371-373, 2002.

[36] E. Shamonina, V. A. Kalinin, K. H. Ringhofer, and L. Solymar, "Magnetoinductive waves in one, two, three dimensions," Journal of Applied Physics, vol. 92, no. 10, pp. 6252-6261, 2002.

[37] R. R. A. Syms, E. Shamonina, V. Kalinin, and L. Solymar, "A theory of metamaterials based on periodically loaded 
transmission lines: interaction between magnetoinductive and electromagnetic waves," Journal of Applied Physics, vol. 97, no. 6, Article ID 064909, 2005.

[38] M. J. Freire, R. Marqués, F. Medina, M. A. G. Laso, and F. Martin, "Planar magnetoinductive wave transducers: theory and applications," Applied Physics Letters, vol. 85, no. 19, pp. 4439-4441, 2004.

[39] R. Mongia, I. Bahl, and P. Barthia, RF and Microwave Coupled Line Circuits, Artech House, 1999.

[40] J. Shekel, "Matrix analysis of multi-terminal transducers," Proceedings of the IRE, vol. 42, no. 5, pp. 840-847, 1954.

[41] R. Islam, M. Zedler, and G. V. Eleftheriades, "Modal analysis and wave propagation in finite 2D transmission-line metamaterials," IEEE Transactions on Antennas and Propagation, vol. 59, no. 5, pp. 1562-1570, 2011.

[42] T. Tamir and A. A. Oliner, "Guided complex waves," Proceedings of the IEEE, vol. 110, pp. 310-334, 1963.

[43] L. Su, J. Naqui, J. Mata-Contreras, and F. Martín, "Modeling metamaterial transmission lines loaded with pairs of coupled split-ring resonators," IEEE Antennas and Wireless Propagation Letters, vol. 14, pp. 68-71, 2015.

[44] J. Hong and M. J. Lancaster, Microstrip Filters for RF/Microwave Applications, John Wiley \& Sons, New York, NY, USA, 2001.

[45] R. Marqués, F. Medina, and R. Rafii-El-Idrissi, "Role of bianisotropy in negative permeability and left-handed metamaterials," Physical Review B, vol. 65, no. 14, Article ID 144440, 2002.

[46] J. Naqui, M. Durán-Sindreu, and F. Martín, "Modeling split ring resonator (SRR) and complementary split ring resonator (CSRR) loaded transmission lines exhibiting cross polarization effects," IEEE Antennas and Wireless Propagation Letters, vol. 12, pp. 178-181, 2013.

[47] R. Bojanic, V. Milosevic, B. Jokanovic, F. Medina-Mena, and F. Mesa, "Enhanced modelling of split-ring resonators couplings in printed circuits," IEEE Transactions on Microwave Theory and Techniques, vol. 62, no. 8, pp. 1605-1615, 2014.

[48] J. Machac, J. Zehentner, and M. Blaha, "Coupling of split ring resonators in a mu-negative volumetric metamaterial," in Proceedings of the IEEE MTT-S International Microwave Symposium Digest, pp. 327-330, Atlanta, Ga, USA, June 2008.

[49] J. Naqui, M. Duran-Sindreu, A. Fernandez-Prieto, F. Mesa, F. Medina, and F. Martin, "Multimode propagation and complex waves in CSRR-based transmission-line metamaterials," IEEE Antennas and Wireless Propagation Letters, vol. 11, pp. 1024-1027, 2012.

[50] M. Beruete, F. Falcone, M. J. Freire, R. Marqús, and J. D. Baena, "Electroinductive waves in chains of complementary metamaterial elements," Applied Physics Letters, vol. 88, no. 8, Article ID 083503, 2006. 

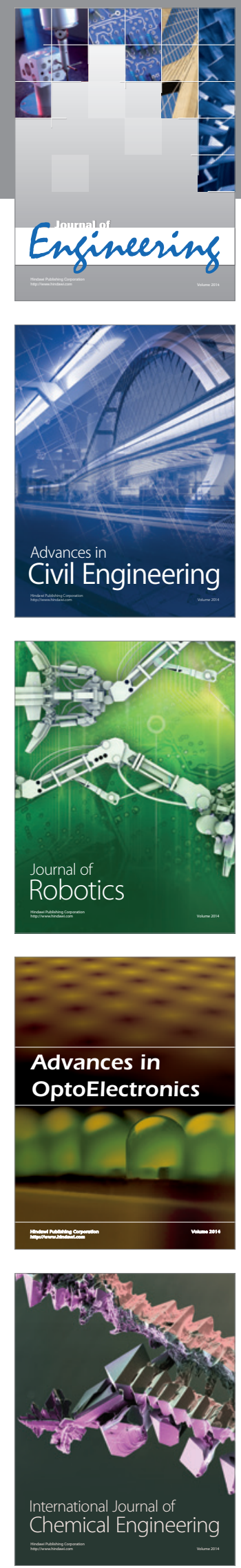

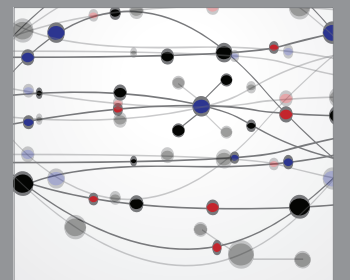

The Scientific World Journal
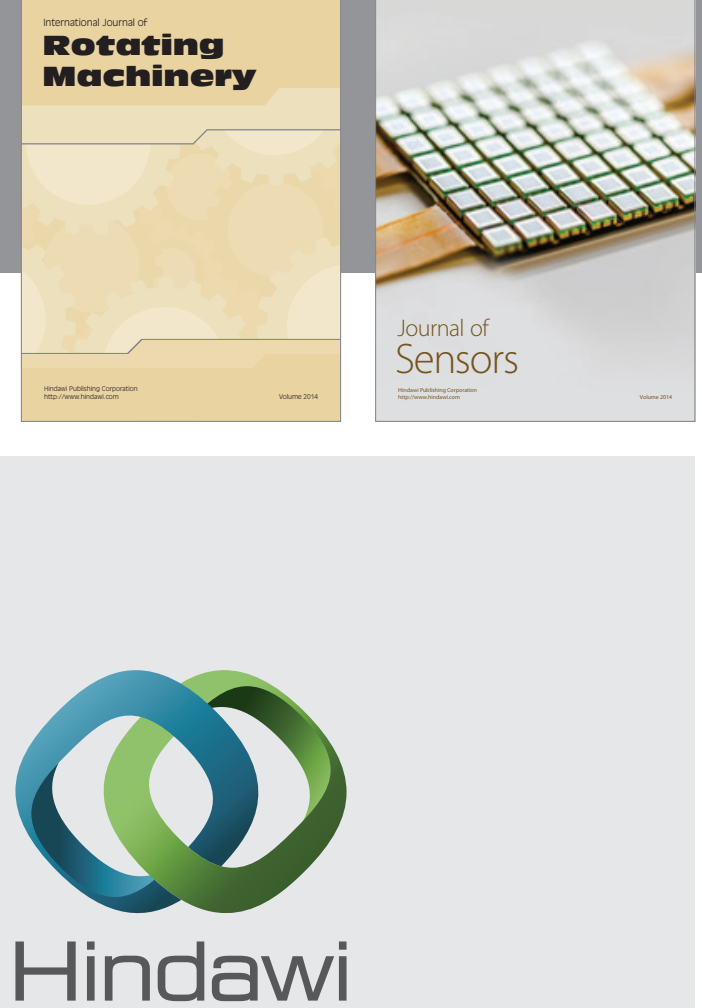

Submit your manuscripts at http://www.hindawi.com
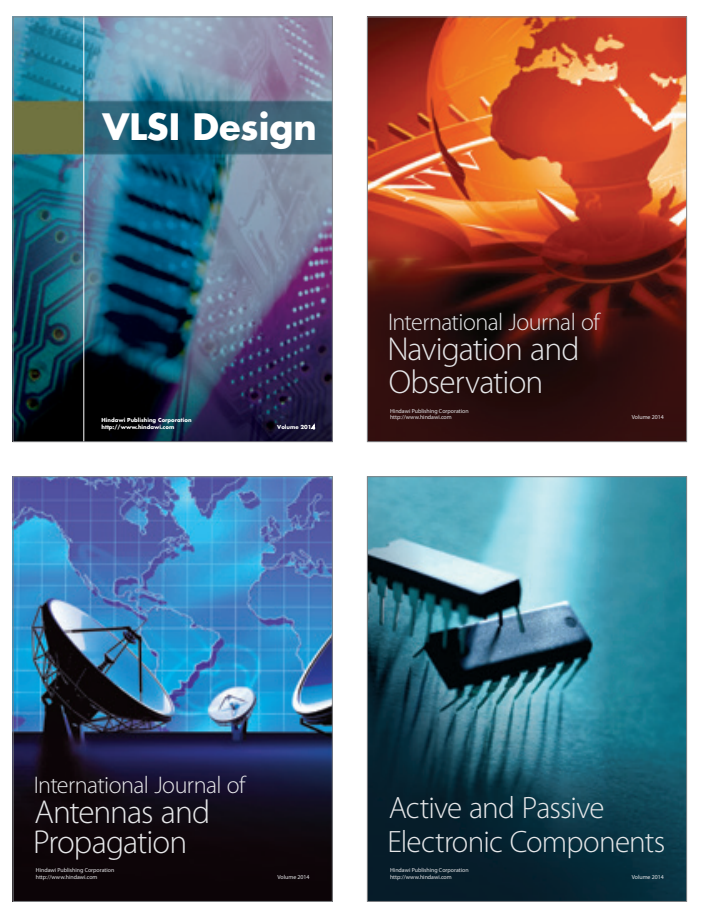
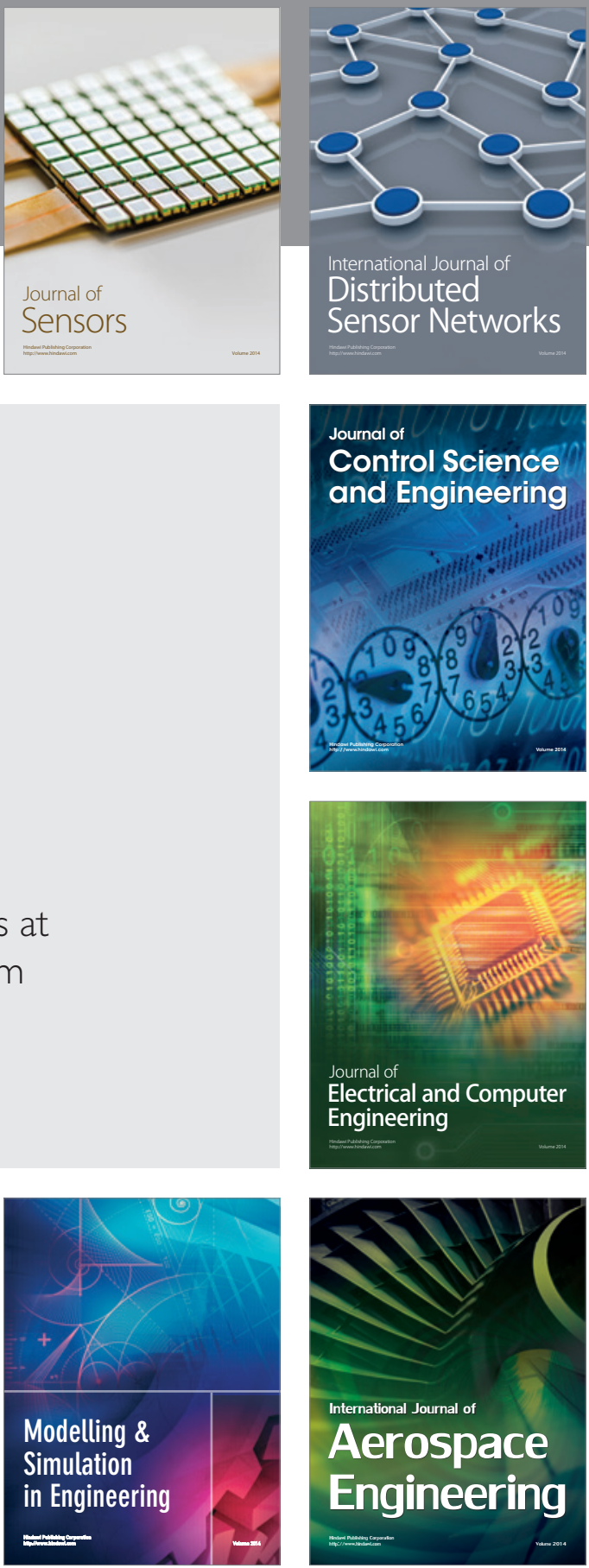

Journal of

Control Science

and Engineering
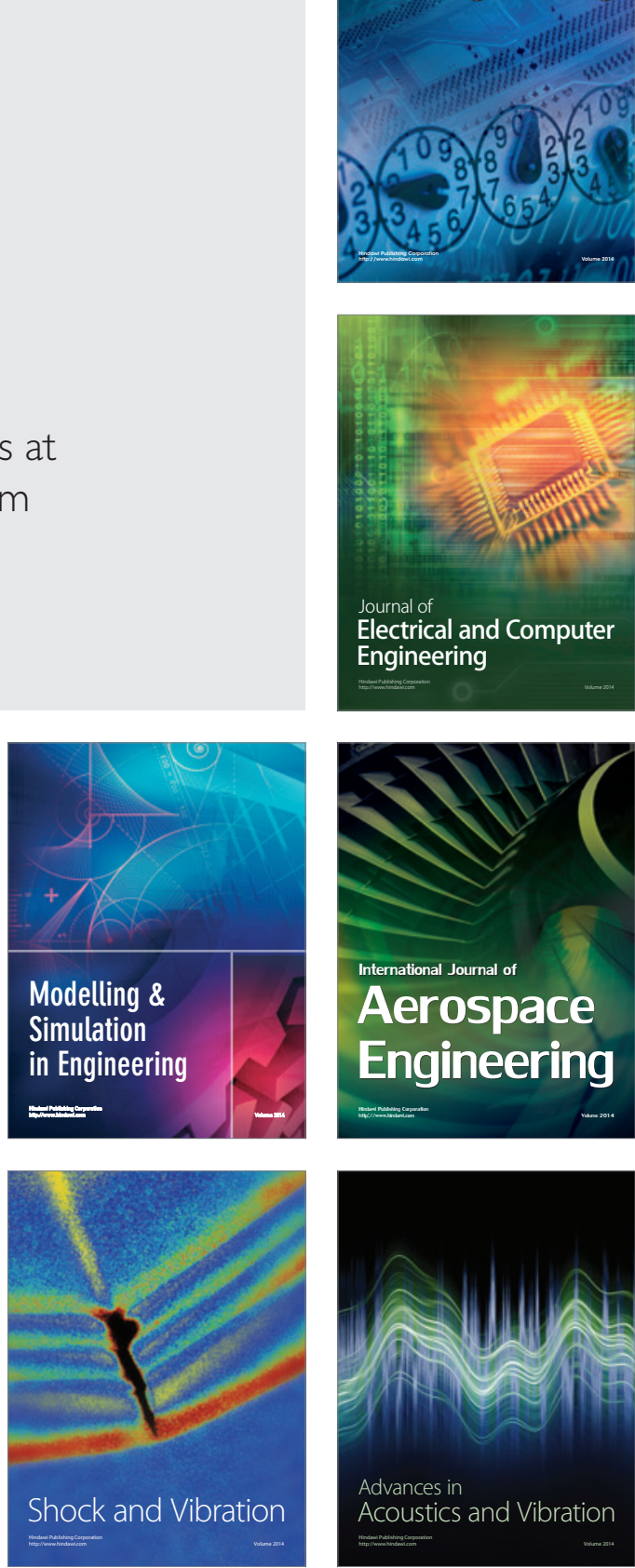